\title{
Expériences du quotidien et sociabilités de migrants roms en squat à l'épreuve du relogement institutionnel
}

\section{Alexandra Clavé-Mercier}

\section{(2) OpenEdition \\ Journals}

Édition électronique

URL : http://journals.openedition.org/pa/400

DOI : $10.4000 /$ pa. 400

ISSN : 2273-0362

Éditeur

Université Lumière Lyon 2

Édition imprimée

Pagination : 101-119

ISBN : 1634-7706

ISSN : 1634-7706

\section{Référence électronique}

Alexandra Clavé-Mercier, « Expériences du quotidien et sociabilités de migrants roms en squat à l'épreuve du relogement institutionnel », Parcours anthropologiques [En ligne], 10 | 2015, mis en ligne le 08 octobre 2015, consulté le 07 mai 2019. URL : http://journals.openedition.org/pa/400 ; DOI : 10.4000/pa.400 


\section{Expériences du quotidien et sociabilités de migrants roms en squat à l'épreuve du relogement institutionnel}

\section{Alexandra Clavé-Mercier}

Université de Bordeaux, LAM

Depuis le début des années 2000, on assiste dans une agglomération de province française à l'arrivée de personnes identifiées comme Roms de Bulgarie $^{1}$. La présence de ces migrants est nettement visible dans l'espace public, par l'habitat en squat notamment. Ces squats, appréhendés de façon négative, sont très rapidement perçus comme un problème, tant par les voisins que par les acteurs institutionnels et politiques. La question du nombre (de squats et d'habitants), de la salubrité voire du danger et de la pollution visuelle sont au cœur des préoccupations communes. Ces squats de migrants sont également appréhendés comme des lieux d'habitat contraint, subis par ceux qui y vivent et nécessairement anomiques. Les migrants roms sont alors perçus tantôt comme des victimes, subissant ce lieu et ces conditions de vie qui lui sont inhérentes, tantôt comme perturbant l'urbanité en y créant des marges visibles. C'est à partir de cette définition de la «question rom » comme problème public (Cefaï, 1996) que les acteurs locaux s'accordent sur la nécessité de trouver politiquement des solutions ${ }^{2}$.

La politique locale spécifique qui voit le jour s'oriente principalement sur le (re)logement, l'objectif premier des acteurs politiques étant l'éradication des squats de migrants roms. Plusieurs actions sont menées en ce sens, dont une Maîtrise d'CEuvre Urbaine et Sociale (MOUS) intitulée "Squats Roms ». Elle vise à résorber les squats en sélectionnant des familles et en les relogeant en parallèle d'un accompagnement social et vers l'emploi. Une association travaillant habituellement auprès des demandeurs d'asile et des réfugiés a été mandatée par la Préfecture pour assurer le pilotage technique de cette MOUS.

1 Cet article s'appuie sur un terrain ethnographique de trois ans (2010-2013) dans une agglomération française pour ma recherche doctorale. Elle visait à articuler l'analyse des expériences migratoires de Roms bulgares à celle des modalités de prise en charge institutionnelle de ces migrants. Une partie du terrain s'est également déroulée en Bulgarie, dans les villes et villages d'origine des migrants rencontrés en France. Environ une dizaine de familles élargies, dont les membres datent leurs premiers séjours en France entre 2007 et 2011, a été suivie de près pour cette recherche.

2 Les premières recherches menées sur les migrations de Roms en France et en Europe soulignent largement la construction de ce fait en problème public, à différentes échelles (voir notamment Cousin, 2011 ; Legros, 2011 ; Legros et Vitale, 2011), mais il convient également de le replacer dans une certaine continuité européenne (Olivera, 2011). 
Une équipe composée d'un coordonnateur et de deux travailleurs sociaux a été constituée. La spécificité centrale de cette politique réside dans le processus de sélection de ces migrants, qui a pour condition et objectif l'intégration, tant au regard de la "volonté » que de la «capacité » des migrants à y parvenir. Concernant les modalités de relogement, les acteurs institutionnels privilégient ce qu'ils nomment le logement «en diffus » : les migrants sont relogés sur la base d'unités familiales restreintes (famille nucléaire), dans des appartements éparpillés dans l'agglomération pour éviter l'effet " ghetto $»^{3}$. Les logements ont des statuts divers, allant du logement d'urgence au logement social en passant par le logement transitoire. Chaque famille suit a priori ces étapes dans son parcours de relogement.

C'est donc le logement qui est pensé par tous les acteurs impliqués dans cette politique comme étant la clef de l'insertion dans la Cité et, plus avant, de l'intégration dans la société, en partant de l'idée communément partagée selon laquelle les squats et bidonvilles de ces migrants sont nécessairement des espaces de vie contraints et anomiques. Comment est vécu ce changement d'habitat par les migrants en question? Au-delà des discours préconçus ou préétablis que peuvent recueillir les professionnels par exemple, l'approche ethnographique permet d'accéder aux vécus intimes de ce changement, tout en le contextualisant dans les vies quotidiennes appréhendées dans la diachronie. Cet article propose donc d'interroger l'habiter de migrants roms bulgares en privilégiant une approche ethnographique des expériences. Il s'agit de comprendre les conditions de l'habiter des Roms bulgares à travers $l^{\prime}$ analyse de leurs expériences du changement dans l'habitat, des changements induits par le relogement institutionnel. Ce faisant, il s'agit de questionner les termes de l'habiter, en s'appuyant sur les questionnements proposés par Florence Bouillon (2009 : 8-9) à partir de la définition de Marc Augé (1992) :

L'habiter est ici envisagé comme la construction d'une relation matérielle et symbolique à l'espace, comme la production d'un lieu anthropologique, chargé d'histoire et d'identité, à partir duquel se construisent des attaches, de la stabilité, de la continuité (Augé, 1992). Et la question devient celle de savoir si, et comment, peut s'organiser un habiter dans un espace domestique caractérisé par la précarité matérielle et la stigmatisation sociale.

Dans un premier temps, je retracerai, dans une approche microsociologique et compréhensive, quelques situations d'interactions entre des agents de la MOUS et des migrants roms bulgares au sujet du relogement qui leur est proposé, afin de mettre en exergue des tensions existantes à ce sujet. Pour les comprendre, je montrerai dans un second temps quelles sont les pratiques de l'habiter de ces Roms bulgares, au regard de leurs vies quotidiennes considérées dans une perspective transnationale. Pour finir, j'analyserai les 
effets du relogement ainsi que les tactiques déployées par ces migrants pour trouver un équilibre entre relogement et insertion.

\section{DES INCOMPRÉHENSIONS ENTRE MIGRANTS ET PROFESSIONNELS AUTOUR DU RELOGEMENT}

Dans le cadre de la MOUS, des logements sont attribués aux migrants sélectionnés en fonction des places disponibles. Les seuls critères pris en compte pour décider du lieu de relogement sont la taille de la famille (qui doit correspondre à la taille du logement) et l'imminence de l'évacuation du squat, lorsque la famille y vit encore. Plusieurs situations observées font état d'une certaine incompréhension entre les acteurs de la MOUS et les migrants au sujet de leur relogement, comme on le voit dans les deux cas suivants :

L'équipe de la MOUS propose à la famille $\mathrm{d}^{\prime} \mathrm{Ani}^{4}$ un relogement dans une maison mise à disposition par la communauté urbaine pour la MOUS. Cette maison se situe dans une commune en moyenne périphérie de l'agglomération, donc assez éloignée des squats dans lesquels se trouvent encore les membres de la famille du couple. Des agents de la MOUS s'aperçoivent assez rapidement qu' Ani et son mari n'y habitent pas, la maison étant tout le temps fermée lorsqu'un des techniciens se rend à proximité pour des interventions. Un jour où je suis dans les locaux de la MOUS, celui-ci remet ce sujet sur la table. Se trame alors une longue partie de rigolade entre les agents autour de l'explication avancée par Ani à la travailleuse sociale sur la raison de son «impossibilité » à vivre dans cette maison. Celle-ci raconte qu'Ani lui aurait longuement expliqué que cette maison était « habitée par des fantômes » et qu'elle ressentait alors une atmosphère mauvaise qui les empêchait d'y habiter. Dès lors, le couple vit dans un autre squat, avec la famille. Ani s'est excusée auprès de la travailleuse sociale du fait qu' " elle ne peut pas » habiter dans cette maison.

Le coordonnateur me raconte quelle fut sa surprise lorsque Stefan a dit qu'il ne voulait pas de la maison à B. " parce qu'il préférait rester au squat, il trouvait ça mieux ». Il poursuit: " [Le responsable] a ouvert des yeux comme ça quand je lui ai dit! Ça, on [ne le] comprend quand même pas! ». M'appuyant sur leurs critères, je rebondis sur l'inscription dans la commune comme un point positif, il réagit en rigolant, sans le prendre au sérieux, en disant que « oui, il a dit des choses comme ça, qu'il voulait rester avec son frère... ! », en balayant l'idée d'un revers de la main, comme si ça lui semblait fantaisiste. ${ }^{5}$

D'après ces propos, il apparait incompréhensible aux acteurs de la MOUS que Stefan préfère rester vivre au squat, auprès de sa famille, plutôt que d'être

4 En raison de l'anonymisation choisie pour rendre compte de cette recherche (localité et acteurs), tous les noms propres sont ici modifiés.

5 Entretien, 26 juin 2012. 
relogé dans une ville à l'autre extrémité de la communauté urbaine. D'une part, cette incompréhension doit être replacée dans son contexte: c'est la première fois que cette explication est formulée directement par un migrant auprès d'un agent du dispositif. Étant donné que ces derniers perçoivent l'action de relogement qu'ils mettent en œuvre comme une forme d'action humanitaire, il paraît donc logique qu'ils aient des difficultés à envisager une forme de réticence de la part des bénéficiaires. D'autre part, on voit à travers ces propos rapportés que ce n'est pas l'argument qui prime mais bien le fait en soi : on peut alors faire l'hypothèse selon laquelle Stefan a fait preuve de méfiance vis-à-vis des acteurs de la MOUS et n'a pas osé se livrer en argumentant son choix, du moins dans un premier temps. En outre, les responsables de la MOUS, en mobilisant dans un second temps seulement l'argument de Stefan, montrent qu'il leur importe peu de saisir les logiques sous-jacentes des migrants, se focalisant plus volontiers sur les résultats escomptés, à savoir l'effectivité du relogement.

Cette incompréhension des migrants roms partagée par nombre d'agents est justifiée par le fait que les Roms n'auraient pas les mêmes « codes ", qu'ils seraient trop différents. L'accompagnement au sein de la MOUS vise précisément à « leur donner les codes de notre société »" Dès lors, les acteurs $\mathrm{du}$ dispositif rationalisent leurs incompréhensions des migrants roms en arguant de la différence intrinsèque de ces derniers plutôt qu'en se questionnant sur leurs logiques. Dans certaines situations, des migrants amorcent des tentatives de négociation avec les agents au sujet du relogement. Parmi l'ensemble des domaines sur lesquels porte la prise en charge par la MOUS, c'est la thématique du logement qui est sujette au plus grand nombre de négociations observées. Celles-ci concernent principalement le lieu de ces logements et la présence des grands-parents dans ces derniers. Le cas de Yanko en est un exemple intéressant.

L'accompagnement de Yanko dans le cadre de la MOUS a été jalonné, comme celui des autres migrants, par plusieurs étapes. Yanko est sélectionné dans la MOUS avec sa femme et ses deux fils, de huit et quatre ans, en février 2012. Au mois d'avril suivant, la famille est relogée dans un logement transitoire (maison de la communauté urbaine) au sein d'une commune voisine de celle où ils ont vécu dans différents squats depuis plusieurs années. Quelques mois plus tard, avant l'été, le couple commence à travailler dans une exploitation agricole; ces emplois ont été trouvés par le biais de la MOUS, faisant employer pour la saison une dizaine de migrants dans cette même structure. Dès le mois de juillet, les travailleuses sociales m'expliquent que Yanko est particulièrement apprécié $\mathrm{du}$ chef d'exploitation, parce $\mathrm{qu}^{\prime}$ " il travaille bien ». La travailleuse sociale en charge de l'insertion professionnelle tente donc de négocier avec ce patron pour obtenir un CDI pour Yanko. À 
cette période, l'équipe de la MOUS envisage le fait que la famille partage son logement transitoire avec une autre famille, qu'elle ne connaît pas. Cette organisation est effective quelques mois plus tard.

Il s'agit donc globalement d'un parcours assez linéaire et ascendant en ce qui concerne les piliers officiels de l'accompagnement des migrants dans le cadre du dispositif. Or, ce n'est pas sur cela que s'appuie Yanko pour effectuer des tentatives de négociation avec les agents de la MOUS au sujet de son relogement.

Yanko a demandé à [la travailleuse sociale] si sa mère pouvait habiter avec eux pour s'occuper des enfants en précisant que c'est elle qui s'en occupe le plus, « comme une maman». [La travailleuse sociale] me dit qu'elle a accepté en leur disant de "ne pas trop le dire», mais qu'elle ne comprend pas car la mère ne travaille pas et donc a du temps. Je lui précise qu' elle travaille, en faisant le geste de la manche. Elle dit «ah oui d'accord! » en levant les yeux, et passe à un autre sujet: Yanko a également demandé que ses enfants continuent à être inscrits à l'école de [la commune sur laquelle se situe le squat dans lequel il vivait] jusqu'à la fin de l'année. Cela, [la travailleuse sociale] l'a accepté de suite, en le comprenant comme étant dans l'intérêt des enfants et, plus avant, comme un signe de «volonté d'intégration $» .7$

Ces tentatives de négociation qu'effectue Yanko au sujet de son relogement ne sont donc pas replacées par la travailleuse sociale de la MOUS dans les cadres de référence de ces migrants. De façon générale, si elles sont acceptées, c'est pour des raisons qui ne relèvent pas d'une démarche de compréhension des migrants, mais plutôt de rationalités propres au dispositif et aux logiques qui le sous-tendent.

Que nous apprennent ces « tactiques » (de Certeau, 1980) que les migrants roms bulgares mettent en œuvre dans leurs relations aux agents du dispositif ? De la même façon que les multiples «initiatives clandestines » des réfugiés dans les camps (Agier, 2003 : 75), considérer ces logiques d'agir comme des marges de manœuvre conduit à se questionner plus profondément sur les expériences que font ces migrants de leur relogement. Comment analyser ces incompréhensions et tentatives de négociation concernant les lieux ou les modalités de relogement? Peut-on y voir des indices des attachements des Roms fondés sur leurs pratiques de l'habiter et plus largement sur leurs rapports à la ville et leurs usages de l'espace ?

7 Entretien, 4 mai 2012. 
VIVRE DANS DES MARGES :

PRATIQUES DE L'HABITER ET RAPPORTS A L'ESPACE

Afin d'étayer cette hypothèse, il est nécessaire de se pencher sur les modes d'habiter des Roms à travers l'analyse des pratiques quotidiennes au sein de leurs quartiers en Bulgarie et des squats en France. Apparaissant a priori tous deux comme des lieux de vie en marge voire des enclaves, il convient d'aller au-delà de cette perception commune en privilégiant une approche de l'intérieur pour comprendre comment les personnes vivent en ces lieux, ce qu'elles font de ces lieux et ce que ces lieux font d'elles.

\section{Habiter dans les mahala: des vies dans l'entre-soi au-delà de l'État}

En Bulgarie, de façon générale, les Roms habitent dans des maisons ou des immeubles au sein de quartiers à part, appelés mahala, dans les villes comme dans les villages. Ces mahala sont géographiquement séparées du village ou de la ville en question par différents symboles matériels : une route, un champ, une ligne de chemin de fer, voire un mur. Selon Alexandra Nacu et Milena Guest (2008 : 107) :

l'appellation de «mahala», terme d'origine turque qui désigne le «quartier», "hameau », renvoie à une entité spatiale composée d'unités sociales monoethniques, qui est aujourd'hui souvent utilisée en bulgare pour désigner un quartier pauvre de mauvaise réputation, suggérant que les habitants sont des Roms.

Les mahala existeraient depuis toujours lorsqu'on pose la question aux habitants. En effet, nombre de ces quartiers existent depuis les années 1930 en lien avec l'exode rural, quand d'autres ont été créés par le régime communiste, pour sédentariser les Roms ou par des opérations d'évacuation urbaine visant à «ne plus avoir de Roms dans la ville ». C'est ce que m'explique Papou Adem, le grand-père de Yanko, âgé de soixante-sept ans. Il ajoute que certains sont ensuite revenus en ville et sont devenus encore plus pauvres, les nouvelles constructions de maisons étant souvent illégales, sans achat de terrain ni permis de construire. À l'heure actuelle, si les mahala citadines sont qualifiées de "ghettos insalubres et surpeuplés » (Ragaru, 1999), toutes se caractérisent par un certain désengagement des pouvoirs publics, que ce soit en matière d'urbanisme, d'éclairage public, de ramassage des ordures et/ou d'entretien des voiries. Mes interlocuteurs se plaisent à répéter $q u^{\prime}$ on sait quand on entre dans une mahala villageoise lorsqu'il n'y a plus de bitume, et dans une mahala citadine lorsque les trous dans l'asphalte sont plus nombreux qu'ailleurs. Ces quartiers ne sont que très peu, voire pas du tout, desservis par les transports publics. De façon générale, tous les services publics (quand ils 
existent en ces lieux), comme les établissements scolaires ou de soins, sont très dégradés et de qualité moindre par rapport à l'ensemble du pays.

Dans le village de Yanko, le maire explique que les 900 Roms se répartissent entre « la grande mahala » où vivent 600 personnes $^{8}$ et deux autres plus petites, qui sont dans le centre du village : l'une «de deux rues » et l'autre « de trois ou quatre maisons $»{ }^{9}$. La grande mahala se trouve à une extrémité du village, séparée de ce dernier par une route nationale. C'est donc la plus visible, mais aussi la plus marquée par la ségrégation de ses habitants, bien que le discours $\mathrm{du}$ maire révèle que le fait que $\mathrm{d}^{\prime}$ autres familles roms vivent au sein même $\mathrm{du}$ village enjoint à qualifier ces micro ensembles de mahala. L'origine « ethnique» des habitants identifiés comme Roms est rapidement mise en avant pour les désigner eux-mêmes mais aussi pour désigner leurs lieux de vie. Cette catégorisation identitaire sous-tend des caractéristiques négatives et induit un agir en conséquence. De façon générale, à part les policiers, aucun habitant du village ne met les pieds dans la grande mahala, tous évitent de traverser les deux autres, préférant faire des détours plutôt que de «passer par chez les Roms ». Ainsi, même sans une situation géographique à la marge, la situation d'isolement est créée de fait par des pratiques d'évitement. De même, les Roms de ce village ne fréquentent pas les cafés qui se situent au centre, ayant dans la mahala des magasins d'alimentation, des bars et des cybercafés. Dès lors, il peut se passer plusieurs jours voire plusieurs semaines sans que les habitants ne sortent de la mahala. Ces sorties sont généralement assez exceptionnelles: pour aller à la poste, au marché, aux bains publics, rendre visite à la famille dans une mahala voisine, ou pour les grandes occasions comme les mariages, baptêmes, anniversaires... La plupart des sorties de la mahala vise à se rendre dans une autre.

À cette ségrégation spatiale doivent être pris en compte d'autres éléments, pour compléter le tableau sur les modes d'habiter de ces Roms. Ces derniers occupent une place équivalente au bas de la hiérarchie sociale du pays, leurs conditions de vie étant marquées par une forte précarité et une exclusion sociale, spatiale et économique. Celles-ci informent nécessairement leurs interactions avec les Bulgares «non-roms ». Se construit alors une expérience subjectivante particulière: en tant que sujets minoritaires mais plus avant, minorisés, toujours renvoyés à leur étrangeté et sans cesse repoussés derrière une frontière (symbolique comme matérielle). Cette frontière multidimensionnelle est au fondement des rapports interethniques comme de leur quotidien dans ce pays.

Ces vies quotidiennes sont donc circonscrites par le territoire de la mahala, ou par un territoire formé par plusieurs mahala géographiquement proches et donc aisément connectées. Les conséquences pour les Roms en termes de

8 Ces chiffres sont ici donnés comme ordre d'idées, m'ayant été communiqués de façon approximative par mon interlocuteur.

9 Entretien, 23 février 2012. 
positionnement dans la société et de rapport aux institutions sont alors indéniables. Cela favorise, par le jeu du cercle vicieux, une dénégation de l'État-nation et un fort repli sur soi, ou plutôt un recentrement dans un entresoi dessiné par les contours de la mahala. On remarque en effet dans les discours des habitants une mise à distance, dans leurs façons de concevoir leurs vies, de tout ce qui est « politique » et de tout ce qui constitue l'armature de la société (désigné par le terme «mafiya»). L'État, en tant qu'instance régulatrice des vies quotidiennes, est absent chez les Roms, qui subissent et construisent cette distance à la fois. Cependant, ils se reconnaissent comme faisant partie de la société bulgare, tout en portant un regard extérieur, détaché de ce qu'il s'y trame, comme si cela ne les concernait pas vraiment, car pas directement. Cette posture est à mettre en lien avec leur place dans la société : ils ne semblent plus rien attendre de l'Etat, ce qui peut s'expliquer par leurs expériences de la scolarité et de l'emploi notamment.

À quoi ressemblent alors ces vies quotidiennes dans l'entre-soi ? La famille est l'institution centrale structurant et régissant les vies des Roms, de par les multiples liens familiaux sans cesse cultivés et réactivés. Elle est au fondement de leur sentiment d'appartenance, de leur quotidien et de l'organisation de l'espace dans les mahala. Les liens interfamiliaux sont cruciaux en termes de vie sociale. Les alliances entre familles, par le biais des mariages, permettent d'assurer ces liens, de les renforcer, ou au contraire de les éviter. Majoritairement, ces mariages ne sont pas contractés officiellement mais uniquement célébrés dans l'entre-soi des mahala, ce qui peut être compris comme un marqueur supplémentaire du rapport distancié des Roms à l'État, perçu ici comme inutile. Cultiver les liens interfamiliaux est donc important dans les vies quotidiennes des Roms bulgares. En outre, les mahala fonctionnant comme un territoire au sein duquel se trame une vie sociale dense, des tensions, des inimitiés voire des rivalités existent. Certaines familles ou personnes ne se côtoient pas particulièrement, quand d'autres ne se parlent pas volontairement. Les raisons peuvent concerner des litiges fonciers, économiques, un manque de respect à une parole donnée, ou un mauvais comportement d'une personne. La stratification sociale et les multiples endoidentifications sont donc très importantes.

Ces dynamiques de sociabilités interfamiliales induisant une hiérarchisation socioéconomique permettent aux Roms de se positionner, de prendre place dans cet entre-soi. Or, les opportunités économiques sont absentes ou extrêmement réduites dans ces vies quotidiennes, ce qui implique des mobilités qui ont cours aujourd'hui vers les pays d'Europe de l'Ouest.

\section{Expériences de vie dans les squats français :} un territoire dans les marges à forte densité sociale

Les squats de migrants roms bulgares sont situés dans des friches industrielles, des zones à l'abandon, situées en marge des villes ou dans ses 
interstices. Vivre en squat recouvre «le fait d'occuper illégalement un lieu privé » (Marpsat, Firdion, 2002: 392) et les conditions de vie y sont très sommaires: des raccordements au réseau électrique sont effectués par les habitants, leur permettant de se chauffer et de cuisiner. Il leur faut s'organiser pour l'accès à un point d'eau, et les sanitaires sont souvent absents ou bricolés par eux.

Une organisation du quotidien propre aux conditions de vie dans les squats se développe, en s'appuyant sur diverses ressources et sur les interactions entre les habitants. Les squats ressemblent à de petits quartiers avec des tâches propres à certains et, à défaut d'institutions, divers espaces collectifs et/ou de socialisation qui ne sont pas sans rappeler l'organisation du quotidien dans les mahala. Il ne s'agit pas là d'un phénomène propre aux Roms, puisqu'on retrouve cette fonctionnalisation des tâches dans l'organisation quotidienne de tout bidonville de migrants, notamment en France après la Seconde Guerre Mondiale (Pétonnet, 1979 ; Sayad, 1995 ; Bernardot, 2008).

Des espaces et des personnes participent à la dynamique du lien social et de la vie collective, celle-ci se déroulant principalement à l'intérieur des squats ou dans le réseau social formé par plusieurs squats. Ainsi il existe toujours un bar clandestin, dans lequel se vendent cafés et sodas. Ceux qui tiennent ces bars sont ceux qui ont le plus de ressources leur permettant de se procurer une machine à café, des gobelets, du sucre et d'investir dans l'achat de produits dans les grandes surfaces avoisinantes. De même, certaines femmes sont des personnes connues par leurs pairs pour vendre des vêtements rapportés des marchés bulgares et diverses marchandises dont le prix et/ou la qualité est préférée en Bulgarie: barres chocolatées, biscuits, chips, teintures pour cheveux, cigarettes. Aux beaux jours, ce type de transaction devient plus visible, les personnes s'installant dehors, devant une petite table garnie, attendant le client (un habitant du squat ou d'un autre squat) en discutant avec d'autres, commentant les derniers faits et gestes des voisins. Des vendeurs ambulants parcourent aussi les squats pour vendre leurs marchandises. Dans certains squats existent également des églises évangéliques ou encore des coiffeuses.

L'organisation matérielle favorisant la débrouille qui prévaut dans ces squats participe donc à reconstruire une vie collective basée sur des éléments rappelant le pays, donc les origines communes, mais aussi sur les liens entre personnes (du même squat ou de différents squats). A travers ces liens circulent les informations, évènements, tout ce qui fonde à la fois la vie dans des maillages très larges d'interconnaissance et dans un espace inconnu, potentiellement dangereux puisque plein d'embûches (administratives, juridiques,...) qu'il s'agit de dompter. Si ce type de recréation d'un entre-soi dans le pays d'accueil est assez classique au début de la migration (Park et al., 1925), la persistance de cet espace de vie ainsi que sa ressemblance avec les mahala est à interroger. Ces deux espaces de vie en marge, mais dans une 
certaine mesure spontanés, sont révélateurs du rapport distancié des Roms à l'État et de l'importance accordée à la vie dans l'entre-soi.

La vie des habitants s'organise spatialement autour d'un ou de plusieurs « nous », reconstituant ainsi dans le squat un espace de l'entre-soi, marqué par de nombreuses distinctions opérées par et entre les personnes en fonction des liens entre elles. L'installation s'effectue auprès des pairs, que sont d'abord les membres de la famille puis les membres de la famille lointaine et les voisins. Le quotidien qui se trame entre les habitants mais également avec les habitants $d^{\prime}$ autres squats se reconnaissant entre eux comme des « semblables » (au-delà des différences de famille, de langue, de village...) est central dans l'expérience des migrants roms. Cette dynamique d'interaction est visible par des multitudes de faits qui fondent le quotidien: discussions, apéritifs, jeux, transactions, mais aussi disputes et conflits ouverts. Elle l'est aussi dans les évènements extraordinaires visant à se positionner dans ces écheveaux interactionnels: fêtes d'anniversaire, de circoncision, de mariage, etc. Il convient en effet de célébrer ces passages dans l'entre-soi sous le regard des semblables afin de signifier son existence, sa valeur, sa richesse.

Le squat, pour ces migrants roms, est donc à envisager comme un espace pour soi, dans l'entre soi, un espace de reconnaissance. Il s'agit là d'une reproduction de la seule forme de vie expérimentée par les Roms dans les mahala, en considérant que l'espace extérieur, en Bulgarie comme en France, est de fait étranger, bien que différent en termes d'opportunités matérielles mais aussi d'interactions. Au-delà du simple logement, c'est la dynamique de l'habiter, intimement liée aux liens interpersonnels pour les Roms, qui est en jeu. Si les squats permettent une organisation spontanée, répondant aux besoins, aux façons de faire et de vivre des Roms, ils favorisent une vie dans l'entre-soi, aux marges de la société, de laquelle l'État est absent. En effet, ces lieux de vie illégaux s'apparentent à des zones de non-droit renforcées par l'illégalité du séjour sur le territoire pour nombre d'habitants et à l'absence de toute intervention étatique dans la vie quotidienne sur ces territoires ${ }^{10}$.

Même si les contraintes (expulsions, langue...) sont nombreuses en France, elles sont contrebalancées par des ressources bien plus importantes qu'en Bulgarie. D'une part, c'est la migration elle-même qui est porteuse de ressources économiques, comme le montrent les activités marchandes déployées au sein des squats et s'appuyant sur le maillage de ces derniers dans la ville. D'autre part, des opportunités économiques autour des squats sont saisies par ces migrants : elles peuvent concerner la ferraille, la mendicité, la prostitution, la construction sur des chantiers, la biffe, les fouilles de

10 Cela est valable au sein des squats uniquement, où les seules interventions sont associatives, l'État et ses représentants n'intervenant la plupart du temps que pour les évacuations des lieux. Toutefois, les habitants de ces squats bénéficient par exemple des services publics que sont les soins et la scolarité, mais qui ne s'inscrivent pas pour autant dans la quotidienneté et/ou le territoire de vie. 
poubelles et containers, l'emploi journalier sur les marchés, etc. De façon générale, les ressources matérielles et immatérielles émergent par le biais de liens interpersonnels (dans ces activités économiques comme dans les dons occasionnels sur les squats ou la chine dans les associations d'aide sociale). De multiples sociabilités au-delà du cercle des pairs se développent donc en France, engendrées en grande partie par l'économie. Ce sont la mobilité géographique et l'habitat en squat qui permettent d'avoir accès à ce type de ressources. La différence entre les deux pays réside en effet dans les opportunités et les interactions qu'offre la ville hors du lieu de vie. L'habiter en squat permet des interactions quotidiennes dans l'entre-soi, mais aussi un ancrage dans un territoire local utilisé au quotidien (commerces, voisins, marché, associations, services sociaux, mendicité, etc.). Cette forme d'habiter, envisagée dans sa totalité (sous un angle matériel et immatériel) permet aux migrants roms de se réaliser dans leur quotidien, au regard des vies fondées sur les liens et interactions dans cet entre-soi permis par le squat, et plus largement par le territoire que forment les squats de migrants roms bulgares. Comme le souligne Alain Tarrius (2001), le lien social fort est un préalable à la réussite économique, ce phénomène renvoyant «davantage à une nouvelle forme d'être collectivement au monde qu'à une façon d'exprimer "à la marge" un processus économique universel ».

Le squat est ainsi l'espace propice à de multiples initiatives informelles et à un quotidien de débrouille, permettant et favorisant des actions quotidiennes relevant d'une certaine addition entre des contraintes exogènes et des rationalités endogènes. Les Roms bulgares envisagent le squat comme espace de vie parce qu'il permet de mettre en ceuvre ces initiatives, de par son caractère de marge et d'entre-soi. Dès lors, ce que l'œil extérieur perçoit comme extrême précarité peut être au contraire appréhendé par les intéressés comme une multiplicité d'initiatives informelles. Cet ensemble contribue à l'usage de la ville que font ces migrants et permet une forme d'insertion bricolée dans et par les marges.

Ces dynamiques de sociabilités, internes dans l'entre-soi des Roms bulgares, comme externes par les liens tissés avec les autres autour (voisins, associations, commerçants...), fondent l'expérience de l'habiter de ces Roms. L'analyse de leurs pratiques quotidiennes a permis de mettre en exergue l'importance des liens, des relations dans l'espace habité, plus que l'espace luimême. Cela fait écho à l'analyse du territoire proposée par Bruno Latour (2010 : 13) en contexte de mondialisation :

Un territoire, c'est d'abord la liste des entités dont on dépend. (...) c'est-à-dire dont le maintien ou l'accès est indispensable à sa propre subsistance (au sens très large et pas simplement alimentaire ou élémentaire du terme). 
Ainsi pour ces personnes, habiter correspond moins à l'attachement à un lieu qu'à un ensemble de liens spatiaux-temporels que l'on peut qualifier de réseaux d'attachements, mêlant affinités territoriales et relations sociales.

\section{UNE MULTIPLICATION DES RESSOURCES DE VIE : ENTRE RELOGEMENT ET INSERTION(S)}

Le relogement institutionnel des migrants roms est encadré par des injonctions plus larges qui visent précisément la rupture de leurs attachements, basés à la fois sur l'informel et sur les liens de sociabilités. Ces derniers se déploient dans un territoire transnational, formé par les interactions soutenues entre des personnes dans les mahala et dans les squats, ainsi que par les nouveaux échanges construits par et dans les marges urbaines. Aussi ces attachements sont mis à mal par la prise en charge de ces migrants dans le dispositif de MOUS visant l'intégration qui s'appuie sur de multiples injonctions comme l'interdiction d'effectuer des séjours en Bulgarie, l'interdiction d'exercer des activités économiques informelles, le relogement en famille nucléaire, l'interdiction d'héberger des proches, etc. « Pour savoir à quoi l'on tient, il faut l'éprouver et le mettre à l'épreuve » souligne Antoine Hennion (2010). C'est précisément le rôle que joue le relogement institutionnel par rapport aux réseaux d'attachement et d'habiter des Roms, comme on le voit précisément en questionnant les effets du relogement sur les intéressés.

\section{L'individuation via le relogement}

Pour les migrants accompagnés dans le dispositif, le relogement fait naître l'expérience concrète de l'individuation, qui est au cœur de la politique d'intégration des Roms. Lorsqu'ils sont relogés, les migrants roms bulgares passent d'un espace de vie spontané, et dans une certaine mesure choisi, à un espace de vie contraint qu'est le relogement institutionnel. De multiples différences existent entre ces types de logement, mais la principale réside dans la solitude ressentie en s'éloignant des pairs et d'une vie quotidienne mêlant la personne au collectif.

C'est notamment l'expérience que fait Shefket, un jeune cousin de Yanko, lorsqu'il est relogé par la MOUS dans un « chalet Emmaüs ». Pourtant, il s'agit d'un lieu de relogement qui est différent de ceux proposés habituellement, plusieurs migrants roms étant regroupés dans ces chalets. Ces derniers se situent dans un quartier excentré de la Ville en voie de réhabilitation. Ils jouxtent un «foyer Emmaüs » dans lequel sont hébergées une vingtaine de personnes. Six chalets côte à côte et distants de quelques mètres sont occupés par des migrants de la MOUS. Lors de sa première visite du chalet avant d'y habiter, Shefket semble percevoir très clairement l'isolement que cela implique : 
Dès notre arrivée, [la travailleuse sociale d'Emmaüs] me laisse avec eux un moment, car elle va saluer le concierge du foyer Emmaüs d'à côté. Très vite, Shefket me dit qu'il est mal à l'aise à cause du silence ambiant, ça semble le choquer vraiment. ${ }^{11}$

Cette première impression est confortée par l'expérience que fait Shefket de ce relogement : il doit vivre seul, ce qui ne lui était jamais arrivé. Cela lui pose des difficultés à la fois pour sa vie quotidienne matérielle (faire à manger, le ménage...) mais aussi en ce qui concerne sa vie sociale : personne à qui parler - à part quelques-uns de ses voisins, mais avec lesquels il n'est pas très proche et qui ne sont pas de sa génération - ni avec qui vivre, plus simplement. C'est de la peur et de l'angoisse qui transparaissent à travers les propos que tient Shefket chaque fois qu'il en parle. Il y passe très peu de temps et me confie ne pas y dormir souvent, ajoutant qu'il n'est pas le seul, comme pour se dédouaner de ce qu'il sait être une «faute » aux yeux des agents du dispositif :

[Il n'y] a rien, là-bas, rien! Alors tout le monde bouge, tout le temps au hangar [squat], avec la famille! ». Je lui demande s'il y dort. Il me répond « oui oui » au début, puis finit par me dire qu'en fait, « pas souvent »... Il m'explique que c'est sa mère qui vient de temps en temps faire le ménage, et que lorsqu'il y dort, il ne fait que ça, il mange toujours au squat : "Juste pour dormir, j'y vais, c'est tout ! ». En riant il ajoute que $c^{\prime}$ est quand même bien quand il veut amener des filles, il est «tranquille ».12

Si Shefket accepte donc d'une certaine façon cette situation pour rester en accompagnement dans la MOUS, il finit un jour par résumer ainsi son expérience de relogement : «Les chalets Emmaüs, c'est la prison pour moi làbas... »13. Ce terme utilisé est fort et peut paraître a priori surprenant lorsqu'on envisage la mahala et le squat comme des lieux de relégation ou d'exclusion, qui seraient donc négativement opposés aux lieux de relogement institutionnels pensés et présentés comme des lieux d'inclusion pour ces migrants. Or, j'ai montré que le squat, comme la mahala, constituent pour les Roms des lieux de vie au sein desquels peuvent s'épanouir leurs liens de sociabilité, et que le squat offre également de nombreuses opportunités pour une « vie bonne » (Butler, 2014). Dès lors, cet espace de vie permet une forme d'insertion dans des marges transnationales s'appuyant sur le «poor-to-poor » (Tarrius, 2015).

Dans ce contexte, l'expérience de Shefket, restituée ici à titre d'exemple, implique un nécessaire renversement de perspective. Ce sont de façon générale les lieux de relogement institutionnels (ces chalets comme les lieux

11 Notes de terrain, 3 avril 2012.

12 Notes de terrain, 24 mai 2012.

13 Notes de terrain, 28 août 2012. 
«en diffus ») qui relèvent d'espaces de vie contraints voire de relégation et de confinement pour les migrants roms, et non les squats. Cela rejoint en partie les conclusions de Marc Bernardot (2005) sur les différentes formes de logement contraint en direction des "populations indésirables » participant à les contrôler, précisément en fabriquant de fait l'individuation concomitante à ce type de logement.

Dès lors, le relogement favorise une sorte de repli sur soi, un isolement des migrants, puisque les logements proposés ne peuvent remplacer l'entre-soi comme cadre protecteur, dans le sens d'espace créateur de sociabilités et d'opportunités. L'individuation induite par la prise en charge de la MOUS a également des conséquences en termes de redéfinition de la famille et des appartenances, créant des frontières entre les migrants sur la base de la sélection et de la « réponse » à l'accompagnement.

L'injonction à l'individuation matérialisée par les modalités de relogement implique pour les migrants roms la naissance de tiraillements identitaires ajoutés à la difficulté de trouver sa place aussi bien dans la société d'accueil (représentée ici par la MOUS) et parmi ses pairs (migrants roms). Une tension entre plusieurs modèles se fait jour puisque l'intégration en question, qui correspond à un modèle d'assimilation, a comme condition une sorte de renoncement : à sa famille, à son organisation de vie quotidienne, soit à ses attachements qui fondent, plus que l'habiter, l'être social, le sujet, dans sa capacité à être acteur (Joas, 1999).

\section{Des tactiques à la « double vie " pour concilier des exigences contradictoires}

Ces effets du relogement, directement vécus ou observés chez des pairs, conduisent certains migrants à développer des tactiques visant à faire avec ce cadre institutionnel sans renoncer pour autant à leurs propres logiques, désirs et rationalités. Les observations effectuées au sujet de Yanko, dont on a vu le parcours d'accompagnement, montrent qu'il se positionne à la frontière entre un quotidien régi par le dispositif et un quotidien dans l'entre-soi des migrants roms. Malgré son accompagnement dans la MOUS, Yanko maintient de forts liens avec sa famille élargie et ses pairs. Au-delà de ces négociations dont j'ai montré un exemple précédemment, Yanko mène ce que l'on pourrait appeler une « double vie » pour concilier certaines injonctions du dispositif de MOUS avec ses volontés quant à l'organisation de sa vie quotidienne.

Quelques jours après avoir appris qu'il est sélectionné dans la MOUS a lieu la fête de la journée des femmes, que les Roms rencontrés sont nombreux à célébrer. Face au malaise provoqué par les décisions de sélection ou de refus et à l'atmosphère tendue qui en découle, Yanko fait le choix de privilégier les liens familiaux en organisant une fête avec nombre de victuailles, boissons et l'accompagnement musical comme il se doit : 
Arrivée au squat de F. vers $14 \mathrm{~h}$, il y a la musique à plein volume. Yanko s'est improvisé DJ pour «fêter la journée des femmes ». Devant la sono, une table avec du champagne et des sodas, ainsi que des gâteaux; une femme me propose un verre, «c'est que pour les femmes » dit-elle. À côté du DJ, des hommes sont regroupés autour d'une table sur laquelle trône une bouteille de whisky. Plus tard, les femmes et les jeunes filles, toutes très maquillées et très bien habillées pour l'occasion, se mettront à danser autour de "leur » table. Le son est vraiment très fort, on s'entend à grand peine. Dès l'arrivée, Stefan me dit que la mairie a été prévenue et qu'elle a donné l'autorisation. Quelques hommes s'inquiètent néanmoins de savoir si le son n'est pas trop fort. La police municipale arrive quelques temps plus tard. Trois agents marchent vers le groupe d'hommes. Au début ils disent « on comprend, vous voulez faire la fête parce que certains d'entre vous ont eu des cartes de séjour ». Yanko s'explique avec eux, en mettant en avant que la mairie est au courant; les policiers le savent, lui disent que [le directeur de cabinet du maire] les a prévenus, qu'il n'y a pas de problème jusqu'à 19h. Yanko s'écrie, «non non jusqu'à $17 \mathrm{~h}$ ! ». Mais le problème soulevé est bien évidemment le volume sonore ; Yanko dit " oui oui on va mettre moins fort, pas de problème, pas de problème ». L'échange est très cordial et dure moins de 5 minutes. Yusuf, le père de Yanko, se moque un peu d'eux en leur disant avec un grand sourire «merci, au revoir!» en français et fait une grimace après, en rigolant. Le volume est alors baissé pendant deux minutes, et à peine la voiture de police repartie, la musique est remise à plein volume. ${ }^{14}$

Le fait que Yanko organise cette fête pour ses proches, sans mettre en avant sa sélection dans la MOUS mais la traditionnelle "journée des femmes », montre qu'il lui importe de se positionner plus dans l'entre-soi des migrants que dans la société française. En outre, son attitude lors de la venue des policiers est significative: parlant français et organisant la fête, il s'institue spontanément porte-parole en se montrant très cordial et bienveillant avec eux. Cependant, c'est lui qui monte de nouveau le volume de la musique lorsque les policiers sont partis, suivant son père qui lui ouvre la voie par ses moqueries des policiers. Dans ce contexte intime du squat, auprès de ses pairs, Yanko privilégie les liens familiaux à son obéissance envers les représentants de l'État que sont ici les agents des forces de l'ordre.

Par ailleurs, dans un premier temps de son accompagnement, Yanko demeure au squat de F. malgré le logement qu'il a obtenu dans le cadre du dispositif. Il ne le verbalise pas aux agents de la MOUS, leur cachant complètement cette situation. Cela lui permet de maintenir les liens avec ses pairs de façon quotidienne et de ne pas être déconnecté de la vie du squat, et plus largement, de la vie dans l'entre-soi des migrants roms bulgares de l'agglomération. Il passe ainsi quelques mois à faire des allers-retours entre la commune dans laquelle se trouve son logement transitoire et celle où se situe le squat, avec ses parents : tantôt une partie de la famille dort dans la maison, 
tantôt dans le squat, tantôt ils se retrouvent tous dans le squat, tantôt tous dans la maison.

Peu de temps avant d'être sélectionné, Yanko me fait part de son nouveau projet: il décide de devenir «DJ», "parce qu'il y a de plus en plus de Bulgares qui [ne] rentrent plus pour se marier $»^{15}$. Il me montre fièrement le camion qu'il a acheté pour y mettre sa sono ainsi que tout le matériel hifi obtenu «avec les Gitans». La raison qu'il donne alors n'est pas principalement économique, mais bien sociale : il s'agit de se positionner tout en étant utile auprès des migrants roms de l'agglomération. Ni son entrée dans la MOUS, ni son nouvel emploi ne modifient ses plans: je le croise régulièrement ayant installé ses enceintes sur d'autres squats de l'agglomération, lors de fêtes d'anniversaire, de mariage. Il me dit que «ça marche bien, les gens sont contents d'avoir un DJ ici, comme en Bulgarie ! ». À plusieurs reprises, je lui demande s'il parvient à jongler facilement entre son travail agricole et celui de DJ sillonnant les squats de l'agglomération : il se dit " fatigué, avec la route tous les jours, et puis entre la maison de B., puis à F., et toutes les places où je vais pour la musique, jamais je m'arrête, je bouge tout le temps! Mais c' est bien comme ça. »16.

Yanko effectue aussi régulièrement des visites dans les squats, y passant du temps. Ainsi il alimente les liens de sociabilité familiaux et interfamiliaux. Dans cette logique, les fêtes habituellement célébrées par eux en Bulgarie sont un moment particulièrement important pour montrer que, malgré son accompagnement dans la MOUS, il est toujours là avec sa famille et toujours le premier partant pour ces célébrations. Cela montre qu'il est central, pour lui, de continuer ainsi à avoir des interactions soutenues avec les autres migrants roms. Ce faisant, Yanko s'inscrit pleinement dans la vie quotidienne de ses pairs comme dans les évènements importants qui la jalonnent. En d'autres termes, il continue de «faire partie de », ce que l'on peut comprendre comme une forme de résistance face à la logique dominante d'individuation portée par le dispositif de MOUS. C'est précisément ce que James Scott (1985) identifie comme étant alors une "arme des faibles", brandie ici contre une vision étatique de l'intégration qui nécessiterait une certaine rupture des attachements.

\section{CONCLUSION}

Par un certain usage de l'espace et grâce à des expériences de l'habiter s'appuyant sur une pratique de la débrouille ainsi que sur des sociabilités multiples et soutenues, les Roms bulgares observés connaissent une forme 
$\mathrm{d}^{\prime}$ insertion dans la ville, par ses marges et de façon informelle. Cette insertion, bricolée mais cependant effective en termes d'opportunités matérielles et immatérielles, est indubitablement considérée comme extrêmement positive et porteuse par ces Roms bulgares qui franchissent ainsi plusieurs frontières (matérielles et symboliques) pour construire de nouveaux espaces de subjectivation.

Or cette insertion peut se trouver a priori contrainte par les modalités de relogement institutionnel concomitant à la politique spécifique d'intégration des migrants roms, précisément parce que ce relogement en soi ne permet pas de nourrir les attachements au fondement des pratiques de l'habiter de ces migrants roms. La prise en compte des subjectivités dans l'analyse de l'expérience de relogement institutionnel que font ces migrants roms bulgares remet donc en cause l'idée de progrès social et d'intégration avancée par les acteurs politiques et institutionnels pour définir la politique menée.

Ainsi l'on peut comprendre les tactiques déployées par les migrants pour intégrer le changement qu'est leur relogement dans leurs vies quotidiennes, effectuant des aménagements nécessaires au regard de leurs pratiques de l'habiter. Mais ces dernières sont aussi nécessairement modifiées par ce changement dans la condition d'habitat qui induit un territoire de vie plus élargi, à d'autres espaces comme à d'autres pratiques d'habiter. Au-delà du fort attachement au squat et aux pairs analysé dans la vie quotidienne en migration, l'habitat en logement individuel devient une ressource nouvelle pour les Roms bulgares. Ce changement, et ses conséquences en termes d'emploi notamment, induit une certaine complexification de leurs territoires de vie et de ressources qui n'est pas négligeable. Plus avant, cette analyse permet de souligner les enjeux et les effets sous-jacents des changements dans les conditions d'habitat urbain sur les personnes qui en font l'expérience, particulièrement en termes de construction de soi comme sujet et de place dans la Cité.

Je tiens à remercier les évaluateurs de la revue ainsi que les coordinatrices de ce dossier pour leurs relectures et remarques qui ont contribué à enrichir ce texte.

\section{BIBLIOGRAPHIE}

Michel AGIER, «La main gauche de l'Empire. Ordre et désordres de l'humanitaire », Multitudes, Vol. 1, n¹1, 2003, pp. 67-77.

Marc AUGÉ, Non-lieux. Introduction à une anthropologie de la surmodernité, Paris, Le Seuil, 1992. 
Marc BERNARDOT, "Déplacer et loger les indésirables, sociologie du logement contraint », Recueil Alexandries, Collections Esquisses, septembre 2005 [en ligne]. URL : http://www.reseau-terra.eu/article337.html

Florence BOUILLON, Le squat: problème social ou lieu d'émancipation ?, Paris, Editions Rue d'Ulm / Presses de l'école normale supérieure, 2011.

Judith BUTLER, Qu'est-ce qu'une vie bonne ? Paris, Payot, 2014.

Daniel CEFAI, « La construction des problèmes publics. Définition de situations dans des arènes publiques », Réseaux, Vol. 14, n75, 1996, pp. 43-66.

Grégoire COUSIN, "L'émergence en Italie d'une "politique tsigane" face aux mobilités roumaines à la fin des années 2000 », Études Tsiganes, n46, 2011, pp. 28-45.

Michel DE CERTEAU, L'invention du quotidien. t. I. Arts de faire, Paris, Gallimard, 1980.

Milena GUEST, «La ruralité des capitales balkaniques. L'exemple de Sofia », Balkanologie, Vol. XII, n², 2003, pp. 127-150.

Antoine HENNION, "Vous avez dit attachements ?... », in Madeleine AKRICH, Yannick BARTHE, Fabian MUNIESA, Philippe MUSTAR (dir.), Débordements: Mélanges offerts à Michel Callon, Paris, Presses des Mines, 2010.

Hans JOAS, La créativité de l'agir. Paris, Editions du Cerf, 1999.

Bruno LATOUR, « La mondialisation fait-elle un monde habitable ? », Territoires 2040, DATAR, 2010, pp. 9-18.

Olivier LEGROS, «Les pouvoirs publics et la "question rom" » en Europe aujourd'hui », Études Tsiganes, n³9-40, 2011, pp. 42-55.

Olivier LEGROS et Tommaso VITALE (dir.), Dossier «Roms migrants en ville. Pratiques et politiques en Italie et en France », Geocarrefour, Vol. 86, n¹, 2011.

Maryse MARPSA et Jean-Marie FIRDION, "Squat», in Marion SEGAUD, Jacques BRUN, Jean-Claude DRIANT (dir.), Dictionnaire de l'habitat et du logement, Paris, Armand Colin, 2002.

Alexandra NACU, Milena GUEST, « Roms en Bulgarie, Roms en Roumanie - quelle intégration ? », Méditerranée, n¹10, 2008, pp. 105-115.

Martin OLIVERA, "La fabrique experte de la "question rom" : multiculturalisme et néolibéralisme imbriqués », La Revue Lignes, Vol. 34, 2011, pp. 104-118.

Robert E. PARK, Ernest W. BURGESS, Roderick D. MAC KENZIE, Louis WIRTH, The City, Chicago, University of Chicago Press, 1925.

Colette PÉTONNET, On est tous dans le brouillard. Ethnologie des banlieues, Paris, Galilée, 1979.

Nadège RAGARU, «Recompositions identitaires chez les musulmans de Bulgarie: entre marqueurs ethniques et religieux », Balkanologie, Vol. III, n¹, 1999 [en ligne]. URL : $\underline{\text { http://balkanologie.revues.org/290 }}$

Abdelmalek SAYAD, Un Nanterre algérien, terre de bidonvilles, Paris, Autrement, Horssérie, n85, 1995.

James SCOTT, Weapons of the Weak. Everyday Forms of Peasant Resistance, Yale, University Press, 1985. 
Alain TARRIUS, "Le lien social fort comme préalable à la réussite économique », Journal des anthropologues, Vol. 84, 2001 [En ligne. URL : http://jda.revues.org/2592

Alain TARRIUS, Etrangers de passage. Poor to poor, peer to peer, La Tour d'Aigues, Editions de l'Aube, 2015.

RÉSUMÉ : Cet article pose la question du modèle d'intégration par le logement en se centrant sur les pratiques quotidiennes des migrants roms bulgares autour de l'habitat. En partant de réactions de migrants suscitées par leur relogement institutionnel dans une agglomération française, sont questionnées les pratiques de l'habiter et les rapports à l'espace observés dans les mahala bulgares et dans les squats français. L'article montre que les dynamiques de sociabilités fondent l'expérience de l'habiter de ces Roms bulgares. Plus que le seul relogement dont ils font l'objet, c'est leur inscription territoriale transnationale dans les marges qui permet une forme d'insertion active.

MOTS-CLÉS : Roms, squats, migrations, habiter, relogement, insertion, ville. 